\title{
Análisis y perspectivas para gestionar el turismo biocultural: una opción para conservar el ecosistema forestal de Temascaltepec
}

\section{Analysis and perspectives to manage biocultural tourism: an option to conserve the forest ecosystem of Temascaltepec}

\author{
Xochitl Jasso Arriagal*
}

'Universidad Autónoma del Estado de México. Centro

Universitario UAEM Temascaltepec, Estado de México, México.

\section{RESUMEN}

El objetivo de la presente investigación fue el análisis transdisciplinario de la viabilidad del turismo biocultural como alternativa para la conservación de la biodiversidad y el bienestar local. Se registró la riqueza natural, cultural y actividades productivas de Temascaltepec. Se aplicó el análisis de componentes principales y la prueba $t$ de Student para variables culturales-demográficas. Se calculó la citación de frecuencia relativa, la ponderación de 19 actividades productivas y el índice de especialización económica. Once pueblos se diferenciaron por poseer diversidad cultural. En cuanto a la riqueza natural, investigaciones previas han registrado 178 especies de aves, 43 especies de hongos comestibles y 45 especies de orquídeas. Se registraron 15 fiestas patronales y 25 productos cultivados y silvestres que forman parte de la dieta local. Se evidenció la preponderancia de avena, aguacate y durazno, así como la cría de bovino, ovino, equino y la extracción de coníferas. El modelo de turismo biocultural debe consistir en la interacción de hechos socializados y el reconocimiento de la otredad en hábitats conservados para la recreación de los sentidos a partir de la observación de paisajes naturales, obras históricas (templos, minas, esculturas prehispánicas), aves, flora y hongos comestibles. Se concluye que el turismo biocultural debe ser planeado bajo la transdisciplina para que sea una alternativa de desarrollo; además, su gestación debe incluir métodos y técnicas ecológicas para la proyección de senderos y rutas integrando actividades productivas locales, fiestas religiosas, recursos naturales y culturales.

PALABRAS CLAVE: actividades locales, aves, hongos, orquídeas, riqueza cultural, turismo bicultural.

\section{ABSTRACT}

The objective of this research was the transdisciplinary analysis of the viability of tourism biocultural as an alternative for the conservation of the biodiversity and local welfare. The natural wealth and the cultural and productive activities of Temascaltepec were registered. The analysis of main components was applied and a Student's t-test for cultural-demographic variables. The relative frequency of citation, the weighting of 19 productive activities and the economic specialization index were calculated. Eleven towns stand out by their cultural diversity. In terms of the natural wealth, prior research has documented 178 species of birds, 43 species of edible fungi and 45 species of orchids. In this case, 15 festivities and 25 products cultivated and wild that are part of the local diet were registered. The preponderance of oats, avocado and peach was evidenced, as well as the breeding of cattle, sheep, horse and extraction of conifers. The model of tourism biocultural should consist of the interaction of socialized facts and the recognition of otherness in habitats preserved for recreation of the senses from the observation of natural landscapes, historical works (temples, mines, pre-Hispanic sculptures), birds, flora and mushrooms. It is concluded that the bio-cultural tourism should be planned under the trans-disciplinarity to make it an alternative development; also, the gestation should include methods and ecological techniques for the projection of trails and routes by integrating local productive activities, religious festivals, natural resources and cultural.

KEYWORDS: local activities, birds, mushrooms, orchids, cultural wealth, biocultural tourism.

\section{INTRODUCCIÓN}

México se caracteriza por ser un país biodiverso (Ceballos et al., 2009), pero aspectos antrópicos pueden comprometer su continuidad (Organización de las Naciones Unidas para la Alimentación y la Agricultura [FAO], 2005; Klenner, Arsenault,
Brockerhoff y Vyse, 2009; Tafoya, 2012; Trombulak et al., 2004). Las investigaciones sobre conservación y manejo de ecosistemas deberían tener un enfoque holístico. El abordar la relación sociedad-naturaleza exige nuevas formas de investigar que 
reclaman un nuevo paradigma capaz de interpretar situaciones físicas, biológicas, sociales y psicológicas que interactúan recíprocamente ante una dinámica compleja (Pérez y Setién, 2008).

Las comunidades ancestrales son dueñas de recursos naturales; además, poseen la riqueza cultural, establecen lazos comunitarios dando lugar al reconocimiento de la otredad y conservan características propias de identidad que sobresalen ante la aldea global (Terer, Muasya, Dahdouh-Guebas, Ndiritu y Triest 2012; López, Shanley y Fantini, 2005; Leff, 2003). La restauración y conservación de los ecosistemas debe partir de la cosmogonía local, a través de principios etnoecológicos de pueblos ancestrales (Toledo, 1992). El complemento entre el conocimiento científico y el tradicional generan modelos de desarrollo sustentable; el punto de partida es analizar el vínculo entre la naturaleza y la cultura (Garibay, 2009).

La riqueza natural se refiere a la capacidad de generación continua de una infinidad de especies y cada una está interconectada como un solo organismo; además, la continuidad de la biodiversidad depende de la asociación ecológica de cada uno de sus elementos. Cuando los ecosistemas presentan reducción de población de especies, se debe a que sus componentes naturales están bajo presión y a que actividades antrópicas han rebasado la capacidad de su regeneración. Las estrategias de restauración deben partir del registro del conocimiento tradicional sobre formas del manejo sustentable de recursos naturales (Pei, Zhang y Huai, 2009; Toledo, 1992). Los pueblos ancestrales han evidenciado la clasificación de ecosistemas a partir de principios etnoecológicos. Por ejemplo, en aldeas ancestrales de China han clasificado su riqueza natural como bosques sagrados, bosques de madera y hongos, bosques comunitarios, bosque de campo santo y bosques de recreación (Jinlong, Renhua y Qiaoyun, 2012).

La riqueza cultural que poseen los pueblos ancestrales es una alternativa para preservar la riqueza natural, porque a partir de características y rasgos de comportamientos aprendidos a lo largo de generaciones establecen pautas de conducta para enfrentar sus problemas y necesidades (Sánchez-Medence y VictorianoRamírez, 2012). Cuando ellos domesticaron plantas y animales aportaron las bases de la dieta más variada de alimentos saludables y el mejor balance nutricional de variedades locales (Onofre y Felicia, 2011). La herencia ancestral de la diversidad de actividades productivas ofrece los medios para alcanzar el bienestar local y, simultáneamente, son idóneas como complemento de actividades recreativas-saludables, como el turismo biocultural, en donde se debería gestionar la interacción sustentable entre naturaleza, cultura, actividades productivas y recreativas.

Ya que el turismo convencional ha generado mayores desigualdades, principalmente socioeconómicas y ecológicas (Ávila y Ávila, 2016; Ojeda, 2014), se propone un turismo alterno como el biocultural, el cual debería consistir en el desarrollo de experiencias de ocio, recreación, entretenimiento y conocimiento a partir de la conservación de recursos naturales, cultura y actividades productivas como una alternativa para lograr el bienestar de comunidades ancestrales. Lohmann y Panosso (2016) investigan al turismo desde el enfoque sociocultural; el punto de partida es el análisis del desplazamiento de persona por diferentes motivos, ya sea por descanso, ocio, placer, negocio, conocimiento, entre otros que, por lo tanto, involucra a todos los sectores productivos, en específico el sector servicios, en su arista del transporte, alojamiento, hospitalidad, bebidas y alimentos y se complementa con el sector primario (agricultura y ganadería); además, mencionan que el desplazamiento de personas origina diversas experiencias e impactos económicos, culturales, sociales y ecológicos. Por lo tanto, antes de impulsar el turismo, en cualquiera de sus facetas se debe establecer reglas para evitar la transgresión cultural, como buenas costumbres, moral, bienestar familiar, conservación del ecosistema y actividades productivas.

\section{MATERIALES Y MÉTODOS}

\section{Área de estudio}

El municipio de Temascaltepec se localiza en el Estado de México, entre las coordenadas geográficas con una longitud mínima de $99^{\circ} 48$ '50" y máxima de $100^{\circ} 14^{\prime} 20^{\prime}$ y con una latitud mínima de $18^{\circ} 58^{\prime} 43^{\prime \prime}$ y máxima de $19^{\circ} 13 ’ 54$ ” (Fig. 1). Presenta varios climas por su diversidad altitudinal. El clima templado subhúmedo $\mathrm{C}(\mathrm{w} 2)$ comprende $54 \%$ del territorio de Temascaltepec; el semicálido tropical lluvioso ACw2 o semicálido subhúmedo, 28\%; el semifrío subhúmedo con lluvias en verano C(E)(w2), 14\%; y el clima tropical lluvioso o cálido subhúmedo con lluvias de verano A(w1), únicamente 2\% (Inegi, 2010). 


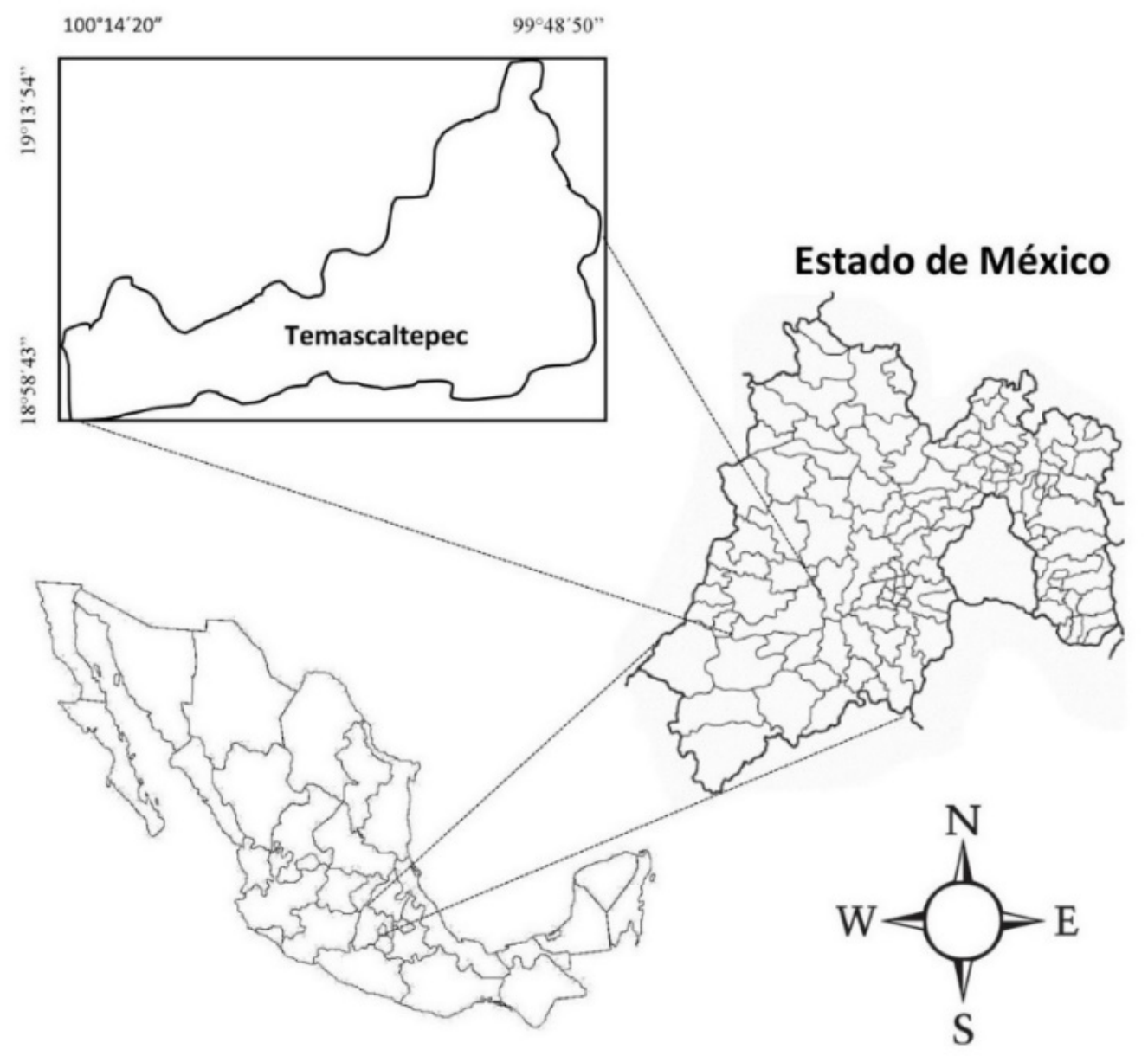

FIgURA 1. Área de estudio.

Fuente: elaboración propia con datos de Inegi.

El estudio se desarrolló bajo el enfoque de la trasndisciplina; partió de la planeación prospectiva, en específico, de la proactividad, la cual consiste en actuar antes de que se agoten los recursos naturales por actividades antrópicas, como son la deforestación, incendios forestales y el cambio de uso forestal. Se utilizó el muestreo no probabilístico intencional para la selección de informantes clave. Se consideró que sean nativos de Temascaltepec, con una edad mínima de 40 años de vivir en el municipio. Se trabajó con 10 personas de siete localidades. Dos informantes de Telpintla, uno de Real de Arriba, uno de San Pedro Tenayac, uno de San Andrés de los Gama, dos de Carboneras, dos de la cabecera municipal y uno de Cieneguillas. A los 10 se les aplicó una entrevista semiestructurada considerando como variables clave: costumbres, tradiciones, leyendas, comida, festividades, actividades productivas, hongos comestibles, quelites (plantas comestibles) y sobre el uso de recursos naturales en general.

Se revisaron tesis de licenciatura sobre listados de flora y fauna para Temascaltepec, para contrastar la información sobre el uso de la especie que se obtuvo de la entrevista semiestructuraday su nombre científico se reconfirmó con las claves taxonómicas.

Las variables culturales-demográficas que proporciona el Instituto Nacional de Estadística y Geografía [Inegi] (2010) fueron consideradas en el análisis de componentes principales (ACP) para las 63 localidades de Temascaltepec, y son: población que reside y nació en la entidad; población que reside y nació en otra entidad; población de más de tres años que habla alguna 
lengua indígena (para el área de estudio es el matlatzinca y náhuatl); población de más de tres años que habla alguna lengua indígena y no habla español y población en hogares censales indígenas. El ACP se utilizó porque reduce la dimensión del conjunto de datos, agrupando los datos en un conjunto de observaciones de variables posiblemente correlacionadas y en otro conjunto de valores las variables sin correlación lineal. Para corroborar la relación de variables se utilizó la distribución $t$ de Student para dos muestras independientes. Se utilizó el software SPSS (Statistical Package for the Social Sciences), versión 19 para calcular el ACP y la $t$ de Student.

Se calculó la citación de frecuencia relativa (CFR), a partir de la fórmula retomada de Signorini, Piredda y Bruschi (2009), para identificar las localidades que se distinguen por poseer cultura ancestral de la agrupación de datos del ACP y $t$ de Student:

$$
\mathrm{CFR}=\frac{\mathrm{FC}}{\mathrm{N}}
$$

donde FC es el número de informantes quienes mencionaron localidades en donde predominan las siguientes variables culturales: idioma que se habla, fiestas patronales o religiosas, zonas arqueológicas, templos del siglo XVI- XVIII y actividad minera; y $\mathrm{N}$ es el número total de informantes.

El municipio de Temascaltepec ha sido minero desde la colonia hasta la actualidad, por lo que se calculó el coeficiente de especialización para el subsector 23 extracción de minerales metálicos:

$$
I E E=\frac{e i / e t}{E i / E t}
$$

donde:

IEE = índice de especialización económica de la actividad ei $=$ actividad que se está midiendo en la industria i del municipio et $=$ actividad que se está midiendo en el total del municipio $\mathrm{Ei}=$ actividad que se está midiendo en la industria i de la entidad $\mathrm{Et}=$ actividad que se está midiendo en el total de la entidad Temascaltepec pertenece a la región IV del Estado de México y se caracteriza por el predominio de actividades agropecuarias, por lo que se calculó la ponderación de 19 productos cultivados comestibles de Temascaltepec. Se consideró dicha característica socioeconómica a partir del Código Ético Mundial para el Turismo (Organización Mundial del Turismo [OMT], 1999), artículo 3, donde se menciona que el turismo es factor de desarrollo sostenible y en el apartado 3 dice: “se procurará distribuir en el tiempo y en el espacio los movimientos de turistas y visitantes, con el fin de reducir la presión en el medio ambiente y de aumentar sus efectos positivos en la economía local".

Se realizó una visita al tianguis de Temascaltepec para identificar hongos y quelites que son consumidos, pero no se encontró ninguna especie, así que por medio de ejemplares deshidratados y con fotografías se mostraron a los informantes clave para preguntarles si era la especie que ellos citan y que consumen. El género y algunas especies de hongos y quelites coincidieron con especies que fueron determinadas en la tesis doctoral de Jasso (2013). Para determinar el nombre científico del ave que ellos mencionaron, en campo fue visualizada en compañía de los informantes clave, se tomaron fotografías y consultó la investigación de Gómez de Silva (1997) para identificar el nombre científico.

\section{RESULTADOS Y DISCUSIÓN}

El municipio de Temascaltepec presenta un alto grado de marginación (Consejo Nacional de Población [Conapo], 2010). La migración es alta (PDM, 2012) y se debe principalmente a la falta de fuentes de empleo y a que el sector primario y el comercio-servicios no abastecen la demanda. La población económicamente activa (PEA) joven migra principalmente hacia Estados Unidos a la edad promedio de 14 años. Anteriormente la actividad minera generaba los empleos. En la actualidad solo hay siete minas de las 62 que existían en el siglo pasado (PDM, 2012). El índice de especialidad económica (IEE) para el subsector 23 extracción de minerales metálicos es de 9.8, lo que indica que el municipio está especializado en dicha actividad económica. Sin embargo, no se refleja en la oferta de empleo y bienestar para los temascaltenses, ya que la mayor parte de los mineros son originarios de otros estados de la república mexicana.

Temascaltepec se distingue porque $68.61 \%$ de su territorio tiene abundante vegetación; predominando los bosques mixtos y de coníferas especialmente de Abies religiosa, Pinus spp. y Quercus spp. (Pérez-Silva, Herrera y Ocampo, 2011). Los informantes clave mencionaron especies silvestres con varios usos. En 
específico mencionaron el consumo de chachalacas (Ortalis poliocephala) y tórtolas, siendo la primera una especie endémica. Gómez de Silva (1997) ha registrado 178 aves para Temascaltepec, entre ellas O. poliocephala. También se documentó la caza y consumo de ardilla y armadillo.

Es complicado modificar la dieta de la población local a base de especies silvestres, pero se debe analizar alternativas para conservar dichas especies. Pérez-Silva et al. (2011) hallaron 43 especies de hongos como nuevos registros para el municipio. En las entrevistas se registró el consumo de 14 especies: hongo azul (Lactarius indigo), amarillos o tecomate (Amanita sp.), patitas (Ramaria spp.), clavos o de montón (Lyophyllum spp.), ardillitos (Russula spp.), orejas de puerco (sin referencia), oreja enchilada (Hypomyces sp.), cazahuate (Pleurotus sp.), hongo de leche (Lactarius sp.), hongo de llano (sin referencia), orejas enchiladas (Lactarius deliciosus), hongo de venado (Hydmum sp.), flor de calabaza (Cantharellus sp.) y gachupines (Helvella spp). Además, Morales (2006) encontró 45 especies de orquídeas, clasificadas en 22 epífitas, 19 terrestres, tres epífita-terrestre y una litofita-epífita. Las aves, hongos y orquídeas son la base para gestionar el turismo biocultural, concebido como un hecho socializado en donde se crisola la expresión de conocimientos tradicionales de especies útiles en hábitats conservados para el deleite de los sentidos y del ego ecológico de personas cultas, altruistas, ecólogos y amantes de la naturaleza.

Valdez y López (2003) registraron Trema micrantha (L.) Blume para Temascaltepec, de dicha especie se extrae el papel amate (López, 2005). Antes de la colonización se usaba en trajes ceremoniales y en ofrendas a los dioses. Los libros prehispánicos y códices también estaban hechos de papel amate. Hoy en día, es insumo para hacer artesanías. Este es el perfecto recuerdo de viaje por ser ligero, fácil de transportar y más económico que otras artesanías (López, 2005). El municipio al poseer dicha especie tiene la oportunidad de planear estrategias de conservación de $T$. micrantha a través de viveros o propagarla en áreas comunales o ejidales, así como crear talleres de manejo sustentable de dicha especie para hacer somvenirs turísticos.

Serna, Pérez y Cardoso (2010) registraron 48 plantas hospederas para la clase insecta del orden lepidóptero de hábito diurno para Temascaltepec, una de ellas es Bursera bipinnata Engl.; Purata y León (2005) la documentaron su uso en aspectos rituales; su resina es conocida como copal o copalli (vocablo náhuatl) y se quema sobre brasas ardientes en recipientes llamados incensarios o sahumerios. La resina despide grandes cantidades de humo blanco aromático; desde el conocimiento tradicional se dice que limpia-purifica y establece un vínculo con lo sagrado. La especie fue encontrada en Telpintla, localidad de Temascaltepec. No se identificó la extracción de su resina, pero sí el uso ritual, por lo que representa una oportunidad para la gestión de viveros y la extracción sustentable de su resina en zonas comunales y ejidales.

La sociedad contemporánea tiene un modo de vida acelerado y, por consecuencia, cada vez más enfermedades y estrés; además, cada día la mente humana se va quedando sin trabajo intelectual, se han dejado a un lado tradiciones familiares, sociales, genéricas, étnicas y raciales (Pakman, 1994 citado en Morin, 1998). La naturaleza, junto con la cosmovisión de los pueblos ancestrales, ofrece alternativas de reencuentro con la vida y de su ser y la desintoxicación del cuerpo (Toledo, 1992; Garibay, 2009); por ejemplo, el temascal practicado en el municipio por personas adultas, en donde se utiliza la resina de B. bipinnata y plantas medicinales.

En las últimas décadas, el hábitat de las aves, los hongos y las orquídeas ya mencionadas ésta siendo amenazado por actividades antrópicas, porque a finales de abril y principios de mayo de 2017 se propagaron incendios forestales, que acabaron con más de 300 hectáreas de bosque (Miranda, 2017), así como por la deforestación (Guerrero-Flores, Macías-Sánchez, MundoHernández y Méndez-Sánchez, 2013). Montaño (2017) evidencia la deforestación de 2000 hectáreas de bosque de pino. Sin embargo, el ecosistema forestal de Temascaltepec aún es ideal para gestionar el turismo biocultural, en donde se deben planear actividades recreativas ecológicas como la observación de aves (178) y escuchar sus hermosos cantos, el deleite de admirar orquídeas silvestres (45), paisajes, cascadas, ríos, cañones, atardeceres, armadillos, ardillas, tejones, nutrias (Guerrero-Flores et al., 2013) y otros, así como la colecta de hongos comestibles, quelites, frutos silvestres comestibles (sin rebasar su capacidad de regeneración), cantar o escuchar corridos locales, degustar el caldo de la virgen, los lasloyos, el atole de plato, atole de arrayan, escuchar leyendas e historias locales acompañado con observación sideral y sembrar un árbol (especies nativas, como $T$. 
micrantha y B. bipinnata) como parte de la estancia ecológica, desde el turismo biocultural, en Temascaltepec.

La riqueza cultural de Temascaltepec está sufriendo cambios y la migración es una de las variables que está influyendo en la pérdida del conocimiento tradicional. Cuando la población nativa se desvincula de la convivencia continúa con la naturaleza, y gradualmente se va perdiendo la estrecha relación entre el cosmos, el corpus y la praxis (Toledo, 1992; Ramírez, 2007). La población nativa de Temascaltepec está migrando a otras entidades del país (Tabla 1), pero también hacia los Estados Unidos: es inevitable este fenómeno; por lo que se requiere de una revalorización del conocimiento tradicional, los recursos naturales, las actividades productivas y la cultura para gestionar alternativas de bienestar.

TABLA 1. Matriz de componentes rotados.

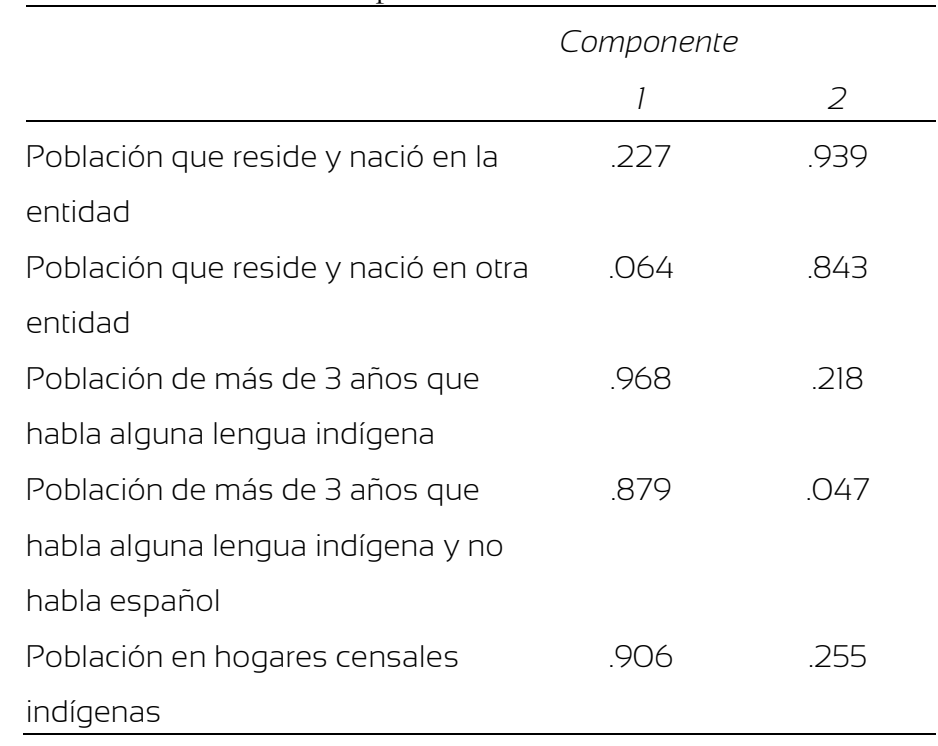

Fuente: elaboración propia a partir de datos de Inegi (2010).

Los resultados de la tabla 1 evidencian que las 63 localidades de Temascaltepec se pueden agrupar en dos grupos. El primer grupo está conformado por la población nativa y habla el matlazinca o náhuatl. El segundo grupo representa a la población inmigrante. Dichos fenómenos permiten interpretar que hay movilidad de población a nivel estatal y municipal, lo que ha dado lugar a que relativamente haya cambio de ideas, costumbres, tradiciones, observaciones, símbolos, creencias, así como en la dieta, vestimenta y lenguaje, ya que antes los hombres vestían con ropa de manta, huaraches y sombrero, y las mujeres de vestido o nagua, sandalias y reboso.

Las 63 localidades de Temascaltepec fueron agrupadas en dos grupos a partir del ACP. El primer grupo está conformado por 21 comunidades con raíces y matices ancestrales. El segundo grupo está conformado por 42 localidades y su población ha dejado de hablar el leguaje nativo y es migrante. La prueba $t$ de Student evidenció que las 21 localidades comparten el lenguaje matlatzinca o náhuatl y solo personas mayores de edad e hijos lo hablan (Tabla 2).

El primer grupo de la prueba $t$ de Student está conformado por 21 localidades y presenta festividades religiosas (Tabla 3) y alimentos típicos (Tabla 4). Sin embargo, los informantes clave remarcaron constantemente que cada vez se están perdiendo y transformando las fiestas, costumbres, valores morales, alimentos y artesanías, como los gabanes de lana que se realizaban en Carboneras.

Las fiestas religiosas pueden ser parte de la programación de actividades de recreación y cultura, en complemento con actividades productivas, como es la floricultura, horticultura y ganadería, que involucre la participación de turistas ya sea en la observación o en la práctica en procesos de siembra, cosecha, ordeña de vacas, elaboración de quesos y crema, elaboración de pan artesanal, nieves y licores, así como en el proceso de producción de productos de café (San Andrés de los Gama).

El maíz, frijol, durazno, aguacate y productos silvestres (Tabla 4) son la base de una dieta diversificada, la cual la hace única y saludable. Además, es una fortaleza alimentaria para la población local y turistas. Las festividades se distinguen por sus colores, sabores, sonidos de vida, alegría y creencias (por ejemplo, la celebración de día de muertos o de los fieles difuntos que se celebra en San Andrés de los Gama); las casas anfitrionas son las que celebran el primer aniversario de fallecimiento de un ser querido. Los familiares, amigos y conocidos llevan el pésame y son recibidos con comida, la cual consiste en una albóndiga de carne rellena con arroz o huevo, que se acompaña con té, café, canela, pozole, tamales y ponche. 
TABLA 2. Prueba de muestras independientes.

\begin{tabular}{|c|c|c|c|c|c|c|c|c|}
\hline & & $F$ & Sig. & $t$ & Gl & $\begin{array}{l}\text { Sig. } \\
\text { (bilateral) }\end{array}$ & $\begin{array}{l}\text { Diferencia } \\
\text { de medias }\end{array}$ & $\begin{array}{l}\text { Error típ. de la } \\
\text { diferencia }\end{array}$ \\
\hline \multirow[t]{2}{*}{ Población que nació en la entidad } & 1 & 22,406 & 0,000 & 5,365 & 62 & 0,000 & 603,192 & 112,441 \\
\hline & 2 & & & 4,261 & 24,304 & 0,000 & 603,192 & 141,547 \\
\hline \multirow[t]{2}{*}{ Población que nació en otra entidad } & 1 & 9,297 & 0,003 & 2,656 & 62 & 0,010 & 19,461 & 7,328 \\
\hline & 2 & & & 1,941 & 21,420 & 0,066 & 19,461 & 10,028 \\
\hline Población de más de 3 años que habla & 1 & 36,563 & 0,000 & 2,827 & 62 & 0,006 & 79,264 & 28,035 \\
\hline alguna lengua indígena & 2 & & & 1,959 & 20,000 & 0,064 & 79,264 & 40,460 \\
\hline Población de más de 3 años que habla & 1 & 9,231 & 0,003 & 1,443 & 62 & 0,154 & 1,000 & 0,693 \\
\hline alguna lengua indígena y no habla español & 2 & & & 1,000 & 20,000 & 0,329 & 1,000 & 1,000 \\
\hline \multirow[t]{2}{*}{ Población en hogares censales indígenas } & 1 & 44,398 & 0,000 & 3,012 & 62 & 0,004 & 171,952 & 57,094 \\
\hline & 2 & & & 2,087 & 20,000 & 0,060 & 171,952 & 82,398 \\
\hline
\end{tabular}

TABLA 3. Calendario de fiestas religiosas mencionadas por los informantes clave.

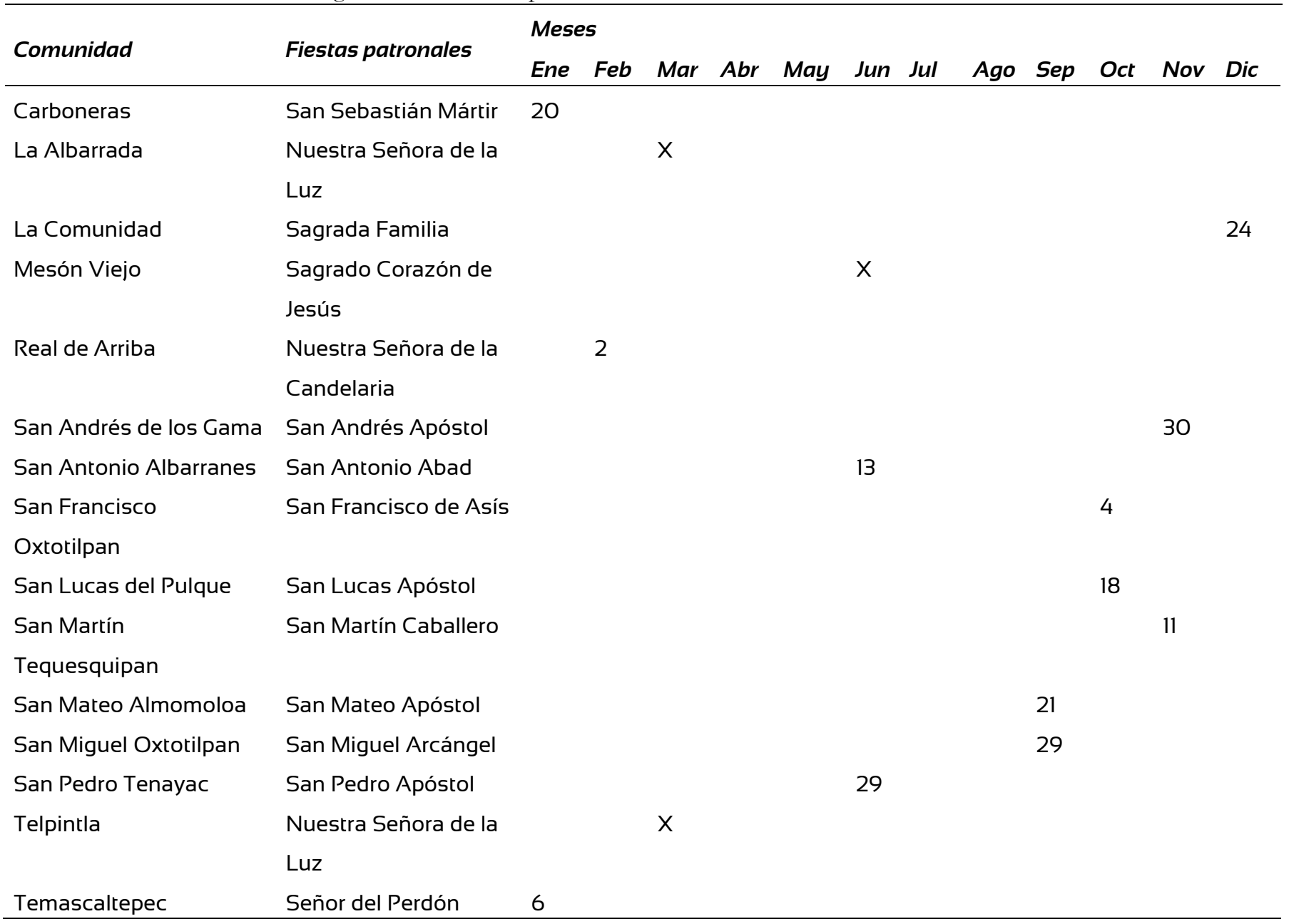


TABLA 4. Productos cultivados, silvestres y transformados.

Meses

\begin{tabular}{|c|c|c|c|c|c|c|c|c|c|c|c|c|c|}
\hline No. & Especies/frutos/alimentos & Ene & $F e b$ & Mar & Abr & May & Jun & Jul & Ago & Sep & Oct & Nov & Dic \\
\hline 1 & Plantas comestibles (quelites) & & & & & & $x$ & $x$ & & & & & \\
\hline 2 & Hongos (comestibles) & & & & & & $x$ & $x$ & $x$ & & & & \\
\hline 4 & Arrayan (amarillo) & & & & & & $x$ & $x$ & & & & & \\
\hline 5 & Guayabas & & & & & & & & & $x$ & $x$ & $\mathrm{x}$ & $\mathrm{X}$ \\
\hline 7 & Peras (Albarranes, San Francisco, Tequesquipan) & & & & & & $x$ & $x$ & $x$ & & & & \\
\hline 8 & Limas (Real de Arriba y cabecera de Temascaltepec) & & & & & & $x$ & $x$ & & & & & \\
\hline 9 & Papas (Mesón viejo) & & & & & & & & & & $x$ & $x$ & \\
\hline 10 & Chícharo & & & & & & $x$ & $x$ & & & & & \\
\hline 13 & Habas & & & & & & $x$ & $x$ & & & & & \\
\hline 14 & Caña (San Pedro Tenayac) & & & & & & & & & & $x$ & $x$ & $x$ \\
\hline 15 & Café (San Andrés de los Gama) & & & $x$ & $x$ & $x$ & & & & & & & \\
\hline 16 & Ciruelas amarilla & & & & & & & & & & $x$ & $x$ & $x$ \\
\hline 17 & Durazno (San Andrés de los Gama) & & & & & $x$ & $x$ & & & & & & \\
\hline 18 & Chile manzano (San Andrés de los Gama) & $x$ & $x$ & $x$ & $x$ & $x$ & $x$ & $x$ & $x$ & $x$ & $x$ & $x$ & $x$ \\
\hline 19 & Nuez (Albarranes) & & & & & & & $x$ & $x$ & $x$ & & & \\
\hline 20 & Higo & & & & & & & & $x$ & $x$ & & & \\
\hline 26 & Licores locales & $x$ & $x$ & $x$ & $x$ & $x$ & $x$ & $x$ & $x$ & $x$ & $x$ & $x$ & $x$ \\
\hline 27 & Atole de plato & & & & & & & & & & & $x$ & \\
\hline 28 & Pulque & $x$ & $x$ & $x$ & $x$ & $x$ & $x$ & $x$ & $x$ & $x$ & $x$ & $x$ & $x$ \\
\hline 29 & Chorreados & & & & & & & & & & & $x$ & $x$ \\
\hline 30 & Chumiates & & & & & & & & & & & $x$ & $x$ \\
\hline 31 & Chínguere & & & & & & & & & & & $x$ & $x$ \\
\hline 32 & Fruta de horno & & & & & & & & & & & & $x$ \\
\hline 33 & Rosquetes & & & & & & & & & & & $x$ & \\
\hline 34 & Pecos & & & & & & & & & & & $x$ & \\
\hline 35 & Lasloyos & $x$ & $x$ & $x$ & $x$ & $x$ & $x$ & $x$ & $x$ & $x$ & $x$ & $x$ & $x$ \\
\hline 36 & Ciciriscos & $x$ & $x$ & $x$ & $x$ & $x$ & $x$ & $x$ & $x$ & $x$ & $x$ & $x$ & $x$ \\
\hline 37 & Bolillo & $x$ & $x$ & $x$ & $x$ & $x$ & $x$ & $x$ & $x$ & $x$ & $x$ & $x$ & $x$ \\
\hline
\end{tabular}




\section{Meses}

No. Especies/frutos/alimentos

Ene Feb Mar Abr May Jun Jul Ago Sep Oct Nov Dic

38 Empanadas: arroz, cajeta, rompope, zarzamora y otros $x \quad x \quad x \quad x \quad x \quad x \quad x \quad x \quad x \quad x \quad x$

39 Pan blanco

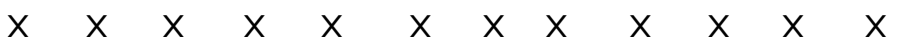

40 La Chimpa (dulce de capulín)

41 Nieve artesanal: zarza, limón, vainilla

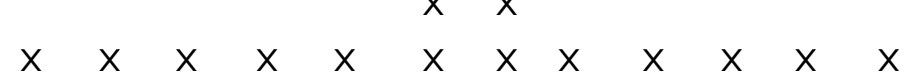

42 Café, dulces y chocolates de café

43 Quesadillas de huitlacoche

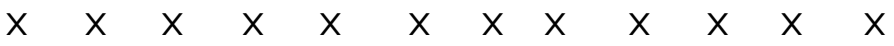

44 Tortas de flor de colorín o zompantle

$x \quad x$

45 Queso y crema de rancho

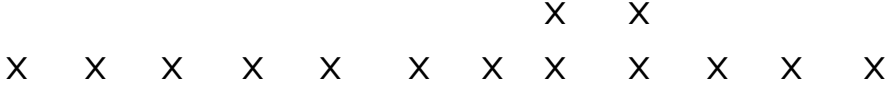

46 Caldo de la virgen

$\mathrm{X}$

TABLA 5. Localidades candidatas para gestionar el turismo biocultural.

\begin{tabular}{|c|c|c|c|c|c|c|}
\hline Localidad & $\begin{array}{l}\text { Personas que } \\
\text { hablan lengua nativa }\end{array}$ & $\begin{array}{l}\text { Fiesta } \\
\text { religiosas }\end{array}$ & $\begin{array}{l}\text { Rastro de zona } \\
\text { arqueológica }\end{array}$ & $\begin{array}{l}\text { Templos: } \\
\text { XVI-XVIII }\end{array}$ & $\begin{array}{l}\text { Fue zona } \\
\text { minera }\end{array}$ & $\begin{array}{l}C F \\
R\end{array}$ \\
\hline Temascaltepec de González & 9 & 10 & 0 & 1 & 0 & 1.1 \\
\hline La Albarrada (San Francisco la & & & & & & \\
\hline Albarrada) & 18 & 1 & 0 & 0 & 8 & 0.9 \\
\hline Mesón Viejo & 7 & 1 & 0 & 0 & 0 & 0.1 \\
\hline Potrero de San José (La Rinconada) & 979 & 0 & 0 & 0 & 0 & 0.5 \\
\hline San Andrés de los Gama & 3 & 5 & 0 & 1 & 0 & 0.6 \\
\hline San Francisco Oxtotilpan & 1223 & 1 & 0 & 0 & 0 & 1.5 \\
\hline San Lucas del Pulque & 17 & 7 & 1 & 0 & 0 & 0.8 \\
\hline San Mateo Almomoloa & 961 & 1 & 0 & 1 & 0 & 0.6 \\
\hline San Miguel Oxtotilpan & 304 & 6 & 0 & 0 & 0 & 0.7 \\
\hline San Pedro Tenayac & 2 & 8 & 0 & 0 & 1 & 0.9 \\
\hline San Martín & & & & & & \\
\hline (Tequesquipan) & 15 & 4 & 0 & 1 & 0 & 0.5 \\
\hline
\end{tabular}

Fuente: Inegi (2010) y trabajo de campo

La tabla 5 presenta las comunidades que pertenecen al grupo uno del ACP y $t$ de Student, además, tienen la CFR con mayor puntaje de acuerdo con las variables culturales que presentan. Las 11 comunidades se pueden dividir en dos grupos. El primero estaría conformado por San Francisco Oxtotilpan, Temascaltepec (cabecera municipal), La Albarrada, San Pedro Tenayac, San Lucas del Pulque, San Andrés de los Gama, San Miguel Oxtotilpan y San Mateo Almomoloa. Las actividades a desarrollar se inscribirían en una ruta denominada: "Encuentro con la naturaleza y cultura"; se realizarían actividades de recreación a partir de la observación de aves, hongos, orquídeas, paisajes de café, durazno, aguacate y bosques, restos arqueológicos y cascos de haciendas. El segundo grupo estaría conformado por La Rinconada, San Martín Tequesquipan y el Mesón Viejo, y sería denominado: "Micoturismo de altura y quelites". Los platillos a implementar serían a partir de hongos y quelites, como los hongos azules (Lactarius indigo), amarillos o tecomate (Amanita sp.), patitas (Ramaria spp.), clavos o de montón (Lyophyllum spp.), ardillitos (Russula spp.), orejas de puerco (sin referencia), oreja enchilada (Hypomyces sp.), cazahuate (Pleurotus sp.), hongo de leche 
(Lactarius sp.), hongo de llano (sin referencia), orejas enchiladas (Lactarius deliciosus), hongo de venado (Hydmum sp.) flor de calabaza (Cantharellus sp.) y gachupines (Helvella spp). En cuanto a los quelites, algunos son: los quintoniles (Amaranthus spp.), choahul (chía roja, sin referencia), cenizo (Chenopodium spp.), nabos tiernos (Brassica sp.), acelga silvestre (sin referencia), verdolagas (Portulaca sp.), lengua de vaca o vinagreras (Rumex sp.) y berros (Nasturtium sp.).

La imagen urbana del primer cuadro de la cabecera municipal se caracteriza por las casas son de adobe, teja, madera y herrería. Esta es otra de las fortalezas para la gestión del turismo biocultural, ya que los pueblos del municipio se encuentran integrados al paisaje atípico, porque existen iglesias del siglo XVI y XVIII, restos arqueológicos (Tabla 5) y bosques. Las actividades productivas que se desarrollan en el municipio se han ponderado calculando el porcentaje de participación de los productos del municipio en relación con los del estado en su conjunto: aguacate, durazno, maíz, avena forrajera, bovino, ovino y equino (Tabla 6) están en los primeros lugares a nivel municipal en comparación con el estado y son parte del fortalecimiento del sector servicios, uno de ellos es el turismo. La misma tabla evidencia que la extracción de pino (12.2\%) y oyamel $(14.3 \%)$ es altamente significativa aunque, desde luego, no es la única forma de generar ingresos, y como ya se ha argumentado, que el turismo biocultural es una alternativa viable.

TABLA 6. Productos agrícolas de Temascaltepec.

\begin{tabular}{lllll}
\hline No. & Producto & Estado de México (t o cabezas) & Temascaltepec (t o cabezas) & Ponderación (\%) \\
\hline 1 & Avena forrajera & 125404 & 2453 & 2,0 \\
2 & Cebada & 16349 & 15 & 0,1 \\
3 & Frijol & 11323 & 62 & 0,5 \\
4 & Maíz & 521586 & 5108 & 1,0 \\
5 & Trigo & 24337 & 8,7 & 0,0 \\
6 & Aguacate & 3207 & 82,9 & 2,6 \\
7 & Durazno & 2716 & 32 & 1,2 \\
8 & Bovino & 573823 & 8482 & 1,5 \\
9 & Porcino & 485637 & 3009 & 0,6 \\
10 & Ovino & 517085 & 5785 & 1,1 \\
11 & Caprino & 145384 & 1275 & 0,9 \\
12 & Equino & 248614 & 2780 & 1,1 \\
13 & Aves de corral & 20720510 & 30733 & 0,1 \\
14 & Conejos & 95309 & 579 & 0,6 \\
15 & Colmenas & 38367 & 214 & 0,6 \\
16 & Pino & 125567 & 15370 & 12,2 \\
17 & Oyamel & 98291 & 14056 & 14,3 \\
18 & Encino & 3392 & 27 & 0,8 \\
19 & Aile & 5594 & 17 & 0,3 \\
\hline
\end{tabular}

Fuente: elaboración propia con datos de Inegi (1999). Nota: no se identificaron toneladas por cultivo para $2010,2015$.

El modelo de turismo biocultural debe considerar la complementariedad entre riqueza natural, cultural y actividades productivas, así como amortiguar los impactos ecológicos por medio de la propagación y reforestación de especies forestales nativas, 
endémicas y prioritarias, como T. micrantha y B. bipinnata. Además, debe establecer políticas ecológicas, algunas de ellas son: establecer fechas de senderismos en los 11 pueblos (Tabla 5) en relación con la época de reproducción de la biodiversidad y festividades. También se debe establecer la cantidad permitida de extracción de hongos, quelites, plantas medicinales y otros recursos útiles. Queda prohibido la extracción de especies endémicas, que estén bajo amenaza y protección especial; solo se deben realizar caminatas etnobotánicas y micológicas en zonas permitidas y que no comprometan la biodiversidad local, así como garantizar la regeneración de especies, ya que es el núcleo central del desarrollo sustentable (Ruiz-Rosado, 2006). El turismo biocultural debe tener cuidado con los impactos ecológicos y establecer un plan proactivo, a partir de los siguientes puntos:

\section{Impactos negativos}

- Deforestación para la construcción de infraestructura (carreteras, redes de agua potable, drenaje), equipamientos (hoteles, restaurantes, cafeterías, agencias de viajes, albercas, canchas de tenis, clubs).

- Contaminación de cuerpos de agua por el incremento de desagües de hoteles y restaurantes.

- Incremento de subproductos de pet, plásticos, cartón y vidrio por la actividad turística

- Incremento del $\mathrm{CO}_{2}$ al incrementar el parque vehicular local.

Algunos de los impactos positivos del desarrollo del turismo biocultural son la revalorización y el empoderamiento de recursos naturales, culturales y actividades productivas por parte de la población joven de Temascaltepec, porque son las fortalezas para generar estrategias de desarrollo local ante los efectos de la globalización como es la fragmentación, exclusión y marginación de territorios (Pradilla, 1996; Hiernaux-Nicolas, 1999). Ante esta dinámica expoliadora, urge el diseño de políticas públicas en las que se tome en cuenta a la población local, nuevas epistemologías como los derechos de la naturaleza y el buen vivir, que trastoca la forma en cómo se relaciona el ser humano y la naturaleza (Ávila y Ávila, 2016).

El desarrollo local debe partir de la conservación de la cultura, los recursos naturales y las actividades productivas, y debe reflejarse en el bienestar de la población local (Pei et al., 2009). El reto es complejo, pero las tendencias de la globalización lo son

\section{Actividad proactiva}

- Diseñar un reglamento de construcción ecológico para el municipio de Temascaltepec que prohíba alterar el hábitat de fauna y flora. La imagen urbana debe conservar los materiales de construcción (adobe, teja, madera, herrería). Las redes de alumbrado público, energía eléctrica y teléfono deben ser subterráneos.

- Gestionar baños ecológicos, plantas tratadoras para aguas jabonosas y presas de oxidación.

- Promover bolsas y botellas ecológicas. Separar y reciclar lo orgánico e inorgánico. Se debe implementar la política de nula producción de subproductos inorgánicos.

- Reforestar zonas perturbadas. Fomentar caminatas, uso de bicicletas, carretas y caballos.

aún más. Las culturas deben recuperar y preservar su identidad; al mismo tiempo, deben ser innovadoras y originales (a partir del bagaje del conocimiento tradicional) y deberán ser capaces de responder a las nuevas demandas de recreación-saludable para que sobrevivan ante la totalidad-parcialidad del desarrollismo global (Pradilla, 1996; Hiernaux-Nicolas, 1999). Bajo esta lógica económica surgen nuevos conceptos como la agroecología, agroforestería y agrobiodiversidad, como estrategias ante la defensa territorial, y en la posibilidad de la custodia colectiva del patrimonio biocultural de los pueblos (Ávila y Ávila, 2016).

La gestión del turismo biocultural debe garantizar la interacción de hechos socializados y el reconocimiento de la otredad en hábitats conservados para la recreación de los sentidos, a partir de la recuperación y preservación de actividades productivas como la producción del café, flores de ornato, durazno, aguacate, ganadería y sus derivados (carnes, embutidos, 
lácteos, leche), frutos locales (Tabla 4) y alimentos-bebidas tradicionales y la revalorización de festividades religiosas. Garantizando la interacción con la cosmovisión ecológica por medio de vivencias recreativa-culturales como es la observación de aves endémicas, los paisajes naturales, obras históricas (templos, minas, esculturas prehispánicas), recolección de flora y hongos comestibles en forma ecológicamente responsable, además de crear opciones de senderismos y rutas en relación con actividades productivas locales (Tabla 6), riqueza natural y cultural.

\section{CONCLUSIONES}

El turismo biocultural debe ser planeado bajo el esquema transdisciplinario para que sea una alternativa de desarrollo local expresado en el bienestar familiar (Fig. 2), su gestación debe incluir métodos y técnicas ecológicas para la proyección de senderos y rutas integrando actividades productivas locales, fiestas religiosas, recursos naturales y culturales.

La agricultura y ganadería (Tabla 6) de Temascaltepec son una fortaleza para la generación de alimentos diversificados para el bienestar familiar. Se registraron 15 fiestas religiosas desarrolladas durante el año en diferentes comunidades. El ecosistema de Temascaltepec presenta una diversidad de hongos (43), orquídeas (45) y aves (178). Esta riqueza natural es una oportunidad para generar actividades recreativas-saludables como la observación de la biodiversidad, respetando las áreas restringidas por el grado de complejidad biológica y zonas de restauración a partir de principios etnoecológicos del pueblo matlazinca y náhuatl del municipio.

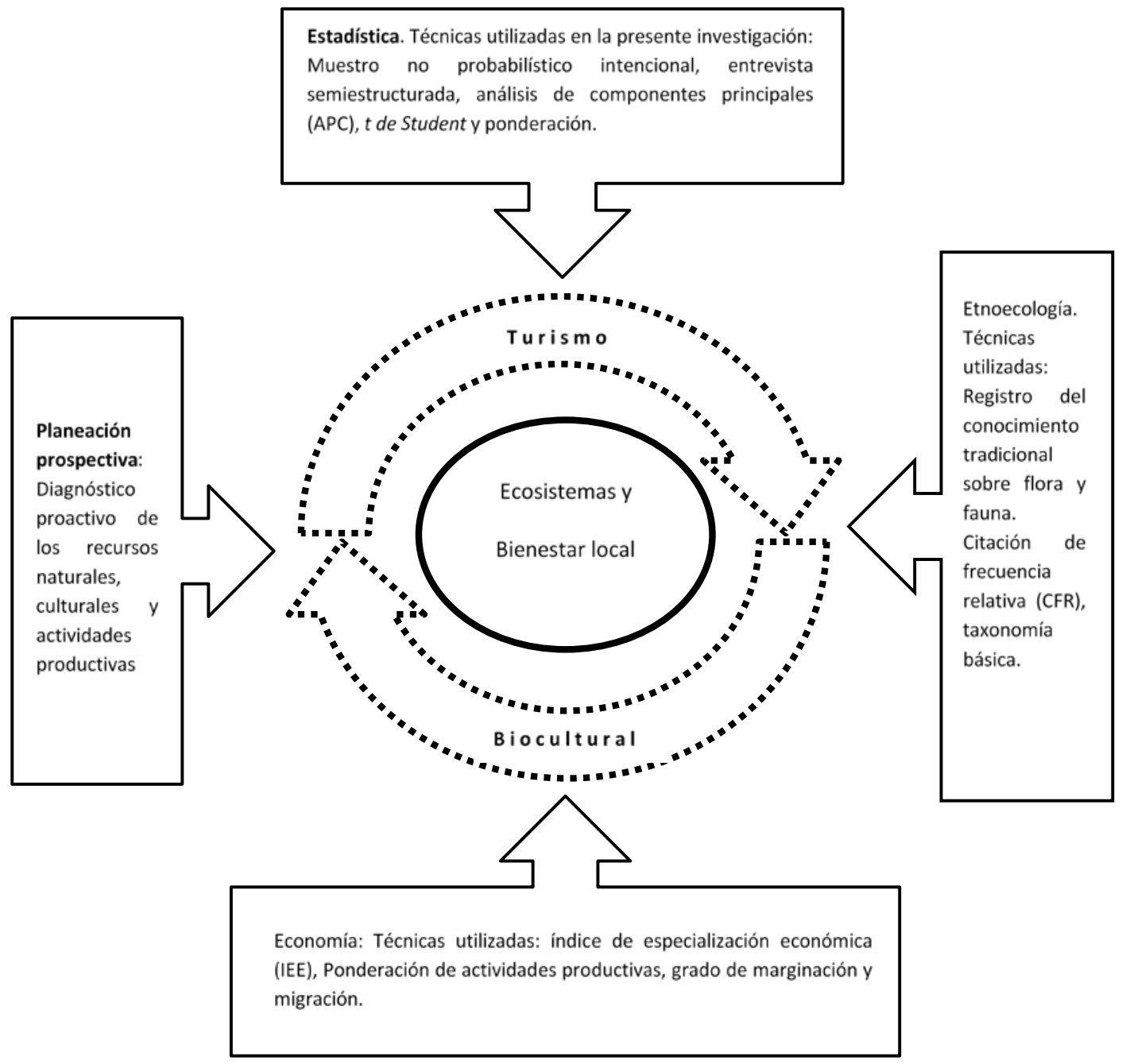

FIGURA 2. Modelo de investigación transdisciplinaria. 
Ante los eventos complejos de la contemporaneidad, los retos sobre el desarrollo local requieren de la integración de variables clave. El turismo biocultural es una actividad antrópica en donde se debe considerar la integración de la riqueza natural y cultural, así como actividades productivas como un sistema complejo, porque cada uno de sus elementos son parte de una red de interacciones que determinan el grado de continuidad de la biota. Es por esto que las investigaciones sobre conservación y manejo de ecosistemas reclaman nuevos paradigmas capaces de interpretar situaciones biológicas, sociales, económicas e ideológicas que interactúan recíprocamente ante una dinámica compleja; en este contexto, disciplinas como la planeación, la estadística, la etnoecología y la economía articuladas bajo la interdisciplina y transdisciplina ofrecen lineamientos para el diseño de modelos de conservación y manejo de la biota.

\section{REFERENCIAS}

Ávila, R. A., \& Ávila R. L. E. (2016). Reconfiguraciones territoriales y neoextractivismo: La nueva zona económica espacial de Chiapas, México. Revista del Departamento de Geografía, 4(7), 4-34.

Ceballos, G., List, R., Garduño, G., Benítez, D. H., Cruz, A. A., \& San Román M., J. E. (comp). (2009). La diversidad biológica del Estado de México. Estudio de estado. México: Conabio.

Instituto Nacional de Estadística y Geografía [Inegi]. (1999). Censo Agricola-Ganadero (Tomo I, II III). México.

Instituto Nacional de Estadística y Geografía [Inegi]. (1995). Anuario Estadístico del estado de México. México.

Instituto Nacional de Estadística y Geografía [Inegi]. (2010). Censos de población y vivienda. México.

Organización Mundial del Turismo [Omt]. (1999). Código Ético Mundial para el Turismo. Recuperado de http://ethics.unwto.org/es/content/codigo-etico-mundial-parael-turismo

Consejo Nacional de Población [Conapo]. (2010). Índice de marginación por localidad a nivel municipal. Recuperado de http://www.conapo.gob.mx/

Organización de las Naciones Unidas para la Alimentación y la Agricultura [FAO]. (2005). Building on gender, agrobiodiversity and local knowledge. Roma, Italia. Recuperado de http://www.fao.org/3/ay5956e.pdf

Garibay-Orijel, R. (2009). Los nombres zapotecos de los hongos. Revista Mexicana de Micología, 30, 43-61. Recuperado de http://museozapoteco.orgfree.com/bibliografia/documento_3.pdf

Gómez de Silva, G. H. (1997). Análisis avifaunístico de Temascaltepec, Estado de México. Anales del Instituto de Biología. Serie Zoología, 68(1), $137-152$

Guerrero-Flores, J. J., Macías-Sánchez, S., Mundo-Hernández, V., \& Méndez-Sánchez, F. (2013). Ecología de la nutria (Lontra longicaudis) en el municipio de Temascaltepec, estado de México: estudio de caso. THERYA, 4(2), 231-242. doi: 10.12933/therya13-127

Heinimann, H. R. (2010). A concept in adaptive ecosystem managementAn engineering perspective. Forest Ecology and Management, (259), 848-856. doi: 10.1016/j.foreco.2009.09.032

Hiernux-Nicolas, D. (1999). Gestión y planificación metropolitana en los tiempos de la globalización: Algunas reflexiones. Quivera, (1), 5358.

Jasso-Arriaga., X. (2013). Un acercamiento al enfoque bolístico: Análisis y perspectivas de los recursos forestales no maderables en una zona natural protegida (Disertación doctoral). Universidad Autónoma del Estado de México, Toluca, Estado de México.

Jinlong, L., Renhua, Z., \& Qiaoyun, Z. (2012). Traditional forest knowledge of the Yi people confronting policy reform and social changes in Yunnan province of China. Forest Policy and Economics, (22), 9-17. doi:10.1016/j.forpol.2011.12.010

Klenner, W., Arsenault, A., Brockerhoff, E. G., \& Vyse, A. (2009). Biodiversity in forest ecosystems and landscapes: A conference to discuss future directions in biodiversity management for sustainable forestry. Forest Ecology and Management, (258S), S1-S4. doi:10.1016/j.foreco.2009.10.037

Leff, E. (2003). La ecología política en América Latina: un campo en construcción. sociedade e Estado, Brasilia, 18(1/2), 17-40. Recuperado de http://www.scielo.br/pdf/se/v18n12/v18n1a02.pdf

Lohmann, G., \& Panosso, N. A. (2016). Tourims theory concepts, models and systems. Boston, Estados Unidos: FAPESP, CABI.

López, C. (2005). Amate: presente de un papel tradicional de corteza. En C. López, P. Shanley, \& A. Fantini (Eds.), La riqueza de los bosques mexicanos: más allá de la madera. Experiencias de comunidades rurales. (pp. 30-35). México: CIFOR/DFID/EC/Overbrook Foundation.

López, C., Shanley, P., \& Fantini, A. (2005). La riqueza de los bosques mexicanos: más allá de la madera. Experiencias de comunidades rurales. México: CIFOR/DFID/EC/Overbrook Foundation.

Miranda, R. (3 de mayo de 2017). Incendios forestales ocasionan catástrofe ecológica en Temascaltepec. El sol de Toluca. Recuperado https://www.elsoldetoluca.com.mx/edomex/incendiosforestales-ocasionan-catastrofe-ecologica-en-temascaltepec

Montaño, M. T. (16 de marzo de 2017). Acusan a minera por ecocidio en Valle de Bravo. El Universal. Recuperado de http://www.eluniversal.com.mx/articulo/metropoli/cdmx/201 7/03/16/acusan-minera-por-ecocidio-en-valle-de-bravo

Morales H., J. L. (2006). Densidad de orquídeas epifitas en el municipio de Temascaltepec, México (Tesis de maestría). Universidad Autónoma del Estado de México, Toluca, Estado de México, México.

Morin, E. (1998). Introducción al pensamiento complejo. España: Gedisa

Ojeda, D. (2014). Paraíso perverso: el turismo como estrategia de conservación neoliberal. El caso del Parque Nacional Natural Tayrona, Colombia. En J. Gascón, \& D. Ojeda (Eds.), Turistas y campesinado. El turismo como vector de cambio de las economias campesinas en la era de la globalización. (pp. 15-39). Madrid, España: Pasos 
Onofre, N. R., \& Felicia, T. D. (2011). Agrobiodiversidad y desarrollo sostenible: la conservación in situ puede asegurar la seguridad alimentaria. Biocenosis, 24 (1-2), 21-29.

Pei, S., Zhang, G., \& Huai, H. (2009). Application of traditional knowledge in forest management: Ethnobotanical indicators of sustainable forest use. Forest Ecology and Management, (257), 20172021. doi:10.1016/j.foreco.2009.01.003

Pérez M., N. E., \& Setién Q., E. (2008). La interdisciplinariedad y transdisciplinariedad en las ciencias: una mirada a la teoría bibliológico-informativa. Acimed, 18(4). Recuperado de http://bvs.sld.cu/revistas/aci/vol18_4_08/aci31008.htm

Pérez-Silva, E., Herrera, T., \& Ocampo, L. A. (2011). Nuevos registros de macromicetos para el municipio de Temascaltepec, Estado de México. Revista mexicana de micología, (34), 23-30

Plan de Desarrollo Municipal [PDM] 2009-2012. Honorable Ayuntamiento de Temascaltepec, Estado de México.

Pradilla, C. E. (1996). Teoría territorial: entre totalización y fragmentación. Revista Ciudades, (29), 15-20.

Purata, S. E., \& León, M. C. (2005). Copal: alimento de dioses y protector de humanos. En C. López, P. Shanley, \& A. Fantini, A. (Eds.), La riqueza de los bosques mexicanos: más allá de la madera. Experiencias de comunidades rurales (pp. 86-91). México: CIFOR/DFID/EC/Overbrook Foundation.

Ramirez, C. R. (2007). Etnobotánica y la Pérdida de Conocimiento Tradicional en el Siglo 21. Ethnobotany Research \& Applications, (5), 241-244. Recuperado www.ethnobotanyjournal.org/vol5/i1547-3465-05-241.pdf

Ruiz-Rosado, O. (2006). Agroecología: una disciplina que tiende a la transdisciplina. Inerciencia, 31(2), 140-145.

Sánchez-Midence, L. A. y Victorino-Ramírez, L. (2012). Guatemala: cultura tradicional y sostenibilidad. Agricultura Sociedad y Desarrollo, (9), 297-313.

Serna T., N. I., Pérez, C. M. A. y Cardoso, J. D. (2010). Plantas hospederas de la clase insecta, orden lepidóptera de bábito diurno, en el parque universitario las orquideas, Temascaltepec, México 2009-2010 (Tesis de licenciatura). Universidad Autónoma del Estado de México, Temascaltepec, Estado de México, México.

Signorini, M. A., Piredda, M. y Bruschi, P. (2009). Plants and traditional knowledge: An ethnobotanical investigation on Monte Ortobene (Nuoro, Sardinia). Journal of Ethnobiology and Ethnomedicine, 5(6). doi: 10.1186/1746-4269-5-6
Tafoya, E. (2012). Implicaciones de la tecnociencia en la modernidad reflexiva. Complejidad, riesgo y democracia. Mundo Nano, 5(1), 17-41. Recuperado de http://www.revistas.unam.mx/index.php/nano/article/view/45 124

Terer, T. A., Muasya, M., Dahdouh-Guebas, F., Ndiritu, G. G. y Triest, L. (2012). Integrating local ecological knowledge and management practices of an isolated semi-arid papyrus swamp (Loboi, Kenya) into a wider conservation framework. Journal of Environmental Management, (93), 71-84. doi:10.1016/j.jenvman.2011.08.005

Toledo, V. M. (1992). What is ethnoecology? Origins, scope, and implications of a rising discipline. Etnoecologica, 1, 5-21.

Trombulak, S. C., Omland, K. S., Robinson, J. A., Lusk, J. J., Fleischner, T. L., Brown, G., \& Domroese, M. (2004). Principles of conservation biology: recommended guidelines for conservation literacy from the education committee of the society for conservation biology. Recuperado de http://www.conbio.org/images/content_prof_dev/conservatio n_literacy_english.pdf.

Valdez P., D. y López, S. J. (2003). Estudio florístico del parque universitario las orquideas en Temascaltepec, estado de México (Tesis de licenciatura). Universidad Autónoma del Estado de México, Toluca, Estado de México.

Manuscrito recibido el: 16 de noviembre de 2016

Aceptado el: 17 de julio de 2017

Publicado el: 23 de marzo de 2018

Este documento se debe citar como:

Jasso A., X. (2018). Análisis y perspectivas para gestionar el turismo biocultural: una opción para conservar el ecosistema forestal de Temascaltepec. Madera y Bosques, 24(1), e2411451. doi: 10.21829/myb.2018.2411451.

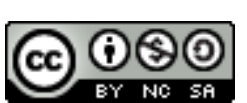

Madera y Bosques por Instituto de Ecología, A.C. se distribuye bajo una Licencia Creative Commons Atribución-NoComercialCompartirlgual 4.0 Internacional. 